\title{
Nachruf Werner Maaßen
}

\author{
In Memoriam Werner Maaßen
}

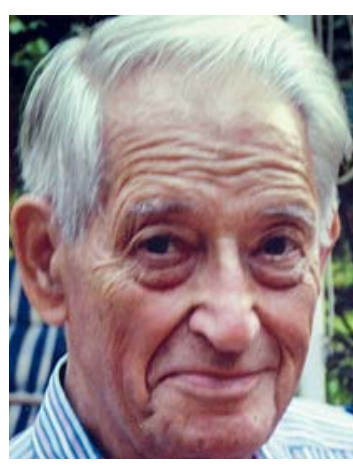

Prof. Dr. med. Werner Maaßen im Jahr 2007

\section{Bibliografie}

Dol $10.1055 / \mathrm{s}-0029-1214737$

Pneumologie 2009; 63:

353-354 @ Georg Thieme

Verlag KG Stuttgart · New York ISSN 0934-8387

\section{Korrespondenzadresse}

Prof. Dr. med.

Nikolaus Konietzko

Spillheide 78

45239 Essen

nikolaus.konietzko@t-online.de
Professor Dr. Werner Maaßen ist tot. Er starb am 7. April 2009 im 89. Lebensjahr zu Haus in EssenFischlaken, in Frieden mit sich, seiner Familie und seinen Freunden - und in Würde, so, wie er gelebt hatte.

Mit Werner Maaßen ist einer der letzten von der „alten“ Garde großer deutscher Lungenärzte gegangen, einer, der noch beide Aspekte der Lungenheilkunde beherrschte und sie auch prägte, die Pneumologie und die Thoraxchirurgie.

Dabei wollte der junge Maaßen, am 17. Oktober 1920 in Düsseldorf geboren und dort auch aufgewachsen, eigentlich Germanist werden, wie er mir einmal verriet. Allerdings hätten es die politischen Verhältnisse der 1930er-Jahre ihm, dem engagierten Mitglied der „Bündischen Jugend“ und überzeugten Nazigegner, unmöglich gemacht, das Studium regelrecht zu Ende zu führen. Denn er stand zu dieser Zeit unter Observanz der Gestapo und hatte bereits eine Nacht in deren Gewahrsam verbringen müssen - wegen despektierlicher Äußerungen vor einem Naziplakat. Deshalb wählte er einen weltanschaulich weniger verfänglichen Beruf und entschied sich für das Medizinstudium.

Nach Ableistung des Arbeits- und Wehrdienstes studierte er an den Universitäten Marburg und Bonn sowie an der Medizinischen Akademie seiner Heimatstadt Düsseldorf. Dort legte er auch Staatsexamen und Promotion ab. Es folgten Lehrund Wanderjahre als Pflichtassistent unter renommierten klinischen Lehrern wie Derra, Boden und Trischen. Anschließend wechselte er als Volontärarzt mit einem Monatsgehalt von 60,- DM - davon musste seine damals dreiköpfige Familie leben - an die Heilstätte Holsterhausen unter Lorbacher. 1953 wurde Werner Maaßen dort an der späteren Ruhrlandklinik, Assistenzarzt, kurz darauf Oberarzt und 1967 Chefarzt. Unter seiner Leitung entwickelte sich die Ruhrlandklinik in weniger als zwei Jahrzehnten vom Tuberkulosesanato- rium zum international renommierten Lungenzentrum.

Obwohl Werner Maaßen alle Facetten der Lungenheilkunde kannte und ihre Techniken beherrschte, galt seine Vorliebe doch dem operativen Fach mit seinen präzisen Techniken und überprüfbaren Resultaten. „Gott schütze das ehrbare Handwerk des Chirurgen“, schrieb er einmal als Marginalie auf einen theorielastigen Artikel, bevor er ihn an mich weiterreichte. So lagen denn auch die Schwerpunkte seiner klinischen Tätigkeit in der Einführung neuer bioptischer Techniken wie der chirurgischen Lungen- und Pleurabiopsie, die sich heute noch „nach Maaßen“ benennt, und in der Perfektionierung thoraxchirurgischer Techniken, speziell der Trachealchirurgie. Aber auch bei der Weiterentwicklung der Bronchoskopie und der Mediastinoskopie hat er Maßstäbe gesetzt.

1966 habilitierte sich Werner Maaßen als Externer an der Universität Münster, zu der das Klinikum Essen damals noch gehörte, und wurde der erste Privatdozent für das Fach Pneumologie in der Bundesrepublik Deutschland. Sein wissenschaftliches Oeuvre weist über 100 Publikationen in nationalen und internationalen Zeitschriften und Lehrbücher aus, u.a. das mit seinen japanischen Schülern verfasste Standardwerk, den „Atlas der Thoraxchirurgie“, 1989 erschienen - in Deutsch und Japanisch.

Sowohl die Entwicklung der Thoraxchirurgie als auch der Pneumologie in Deutschland hat Werner Maaßen wesentlich mitgestaltet. Er war wie nur wenige aufgrund seiner breiten Ausbildung und aus eigenem praktischem Tun heraus in der Lage, den Bogen von der Phthisiologie bis hin zur modernen Pneumologie und Thoraxchirurgie zu spannen. Nicht ohne Grund war er Präsident der Deutschen Gesellschaft für Thorax-, Herz- und Gefäßchirurgie und der Deutschen Gesellschaft für Pneumologie und Tuberkulose, wie sie damals 
noch hieß. Als Ausdruck der Wertschätzung seiner wissenschaftlichen und berufspolitischen Verdienste haben ihn zahlreiche wissenschaftliche Gesellschaften in Europa zum Ehrenmitglied und zum korrespondierenden Mitglied ernannt, eine Aufzählung würde den Rahmen sprengen, ebenso die Erwähnung aller präsidialen Ämter, die er in den nahezu 40 Jahren seines beruflichen Wirkens innehatte.

Werner Maaßen hatte schon früh erkannt, dass sich beide Disziplinen, die Pneumologie und die Thoraxchirurgie, mittlerweile so stark weiterentwickelt hatten, dass das gesamte Fach, die Lungenheilkunde, nicht mehr von einer Person allein vertreten werden konnte. Folgerichtig entschied er, „seine Klinik“ neu zu strukturieren. Chirurg und Internist sollten auf Augenhöhe miteinander diskutieren und agieren. Die damit ausgelöste weitere Entwicklung der Ruhrlandklinik und ihre Blüte haben ihn stets mit großer Genugtuung erfüllt, auch in späteren Jahren, nachdem er 1985 den „Stab“ an mich weitergegeben hatte. In jüngster Zeit freute ihn besonders die Integration der Ruhrlandklinik in das Universitätsklinikum Duisburg-Essen, Bestätigung seiner nahezu 40-jährigen beruflichen Arbeit und weitsichtiger Entscheidungen.

Dank seiner körperlichen Fitness - bis ins hohe Alter machte Werner Maaßen Ausritte im Bergischen Land auf „Abilar“, seinem Wallach - und seiner geistigen Frische hat er nach der Pensionierung sein Leben aktiv und in heiterer Altersweisheit gestaltet. Der frühe Tod seiner Frau Hildegard freilich warf einen langen Schatten auf sein späteres Leben. Doch seine Töchter und Enkelkinder, Freunde, Bücher und Bilder - seine Kirchners, Klees und Miros - haben ihm über manche dunkle Stunde hinweggeholfen. Auf seinen Grabstein hatte Werner Maaßen schon vor Jahren die Rilkeverse meißeln lassen:

Ich kreise um Gott, um den uralten Turm, und ich kreise jahrtausendelang; und ich weiß noch nicht: bin ich ein Falke, ein Sturm oder ein großer Gesang.

Ob er's jetzt weiß? 